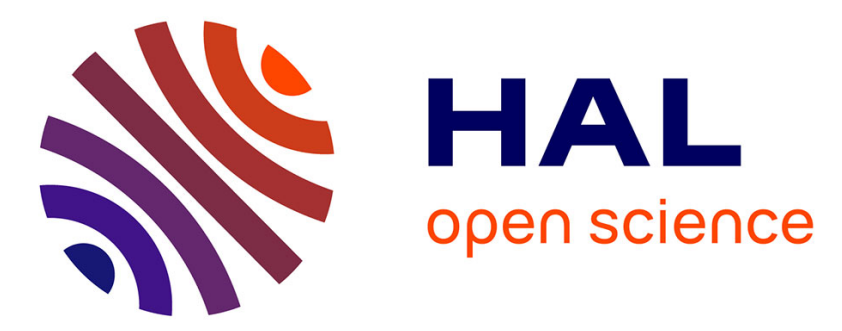

\title{
Gérer en réseau des entreprises de réseau?
}

Anne Marchais-Roubelat

\section{To cite this version:}

Anne Marchais-Roubelat. Gérer en réseau des entreprises de réseau?. Flux - Cahiers scientifiques internationaux Réseaux et territoires, 1998, 14 (33), pp.36-44. 10.3406/flux.1998.1232 . halshs03341244

\section{HAL Id: halshs-03341244 https://shs.hal.science/halshs-03341244}

Submitted on 5 Oct 2021

HAL is a multi-disciplinary open access archive for the deposit and dissemination of scientific research documents, whether they are published or not. The documents may come from teaching and research institutions in France or abroad, or from public or private research centers.
L'archive ouverte pluridisciplinaire HAL, est destinée au dépôt et à la diffusion de documents scientifiques de niveau recherche, publiés ou non, émanant des établissements d'enseignement et de recherche français ou étrangers, des laboratoires publics ou privés. 


\section{Gérer en réseau des entreprises de réseau ?}

Anne Marchais-Roubelat

\section{Citer ce document / Cite this document :}

Marchais-Roubelat Anne. Gérer en réseau des entreprises de réseau ?. In: Flux, n³3, 1998. pp. 36-44;

doi : https://doi.org/10.3406/flux.1998.1232

https://www.persee.fr/doc/flux_1154-2721_1998_num_14_33_1232

Fichier pdf généré le 12/05/2018 


\begin{abstract}
Network operators, or companies which run and manage technical infrastructure to provide services of general utility, are currently in a period of major transition. The question is, are these companies particularly susceptible to being managed like networks? Case studies of this type of management at EDF, the SNCF et the RATP (Électricité de France [electrical power company], Société Nationale des Chemins de Fer [nationalized train system] and Régie Autonome des Transports Parisiens [Paris public transport company]) have shown that, depending on whether or not the dimensions of the enterprise were already aligned with a pre- established set of priorities, the network itself may facilitate the functioning or transformation of the organization. Whether the network would be able to expand, or would have to remain purely local, would then depend more on the strategic importance of the company's functions at any given moment than on the inherent characteristics of the company. Moreover, analysis in terms of functionality shows that a radical transformation of the organizational chart, where a network replaces a pyramid-type diagram, may be more effective in controlling the major functions of the enterprise than making incremental adaptations to local imbalances when they are interdependent. In the first case studied, the network was opposed by its shape to a centralized organization, but maintained the same major functions. In the second case, the combined consequences of imbalances produced network effects which brought the very existence of the company itself into question. A policy of dissociating technical infrastructure management from service management could, in the final analysis, bring about more strategic change over the long run than would simply restructuring the organization or its management.
\end{abstract}

\begin{abstract}
Résumé
Les entreprises de réseau, définies comme des entreprises fournissant des services d'intérêt général à travers l'exploitation d'infrastructures lourdes, connaissent actuellement de profondes mutations. Sontelles alors plus particulièrement susceptibles d'être gérées en réseau ? L'étude de cas de management en réseau à EDF, la SNCF et la RATP montre que selon que ses dimensions sont ou non en phase avec la hiérarchie des priorités déjà établie, le réseau facilite le fonctionnement ou la transformation de l'organisation. L'expansion du réseau ou son caractère purement local dépendrait alors de l'importance stratégique des fonctions qu'il remplit à un moment donné plutôt que de ses caractéristiques intrinsèques. Par ailleurs, l'analyse en termes de fonctions montre qu'une transformation radicale de l'organigramme où la pyramide laisse la place au réseau peut permettre de mieux contrôler les grandes fonctions de l'entreprise qu'une adaptation incrémentale aux déséquilibres locaux lorsque ceux-ci deviennent interdépendants. Dans le premier cas étudié, le réseau s'oppose par sa forme au " château », mais il conserve les mêmes fonctions principales. Dans le deuxième cas, les conséquences croisées des déséquilibres engendrent des effets de réseau susceptibles de remettre en cause l'existence de l'entité elle-même. La question de la dissociation des infrastructures et du service apparaît alors plus porteuse de changements stratégiques à long terme que de spectaculaires remaniements organisationnels et managériaux.
\end{abstract}


Un fonctionnement en réseau

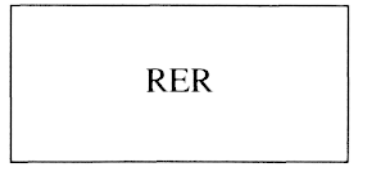

$1 \mathrm{UO}=$

une ligne
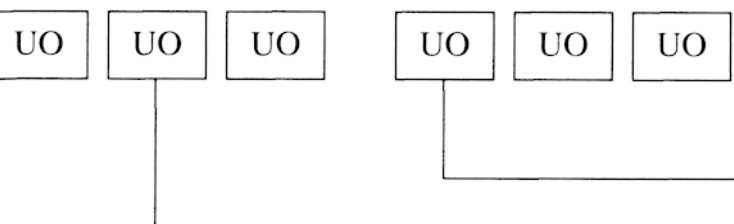

1 centre bus

(10 à 20 lignes)

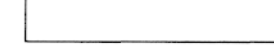

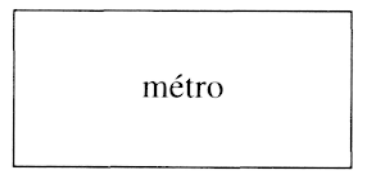

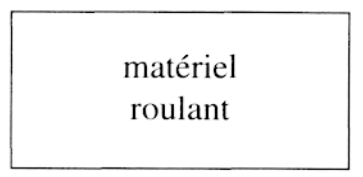

$1 \mathrm{UO}=$

une ligne

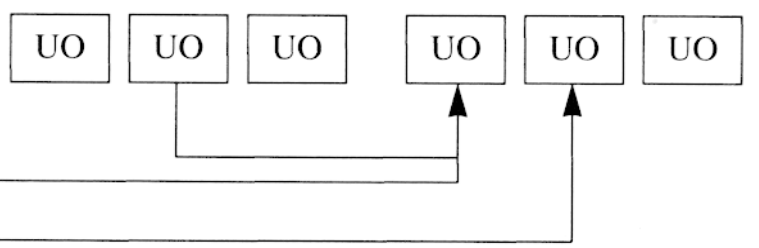

circuits courts

Métro, RER, Bus, sont structurés en Unités Opérationnelles ;

les ateliers sont des unités décentralisées ;

la ligne opérationnelle n'a pas d'atelier en propre, elle signale par un système de dépêches un accident à l'atelier qui le répare.

Figure 1. Unités d'oeuvre à la RATP.

\begin{tabular}{|c|c|}
\hline commercialisation & commercialisation \\
\hline production & production \\
\hline
\end{tabular}

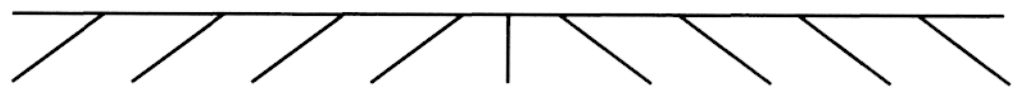

réseau $\mathrm{A}$

réseau $\mathrm{B}$

Figure 2. Fusion de réseau sur la dimension commerciale. 
FLUX

$n^{\circ} 33$

Juillet - Septembre

1998

pp. $36-44$

\section{Gérer en réseau des entreprises de réseau?}

ANNE MARCHAIS-ROUBELAT

Anne MARCHAIS-ROUBELAT est maître de conférences en sciences de gestion au Conservatoire national des arts et métiers. Ses travaux portent sur la phénoménologie de l'action, les processus de changement organisationnel et le management en réseau. Elle a développé un modèle conceptuel qu'elle a appliqué à des actions complexes ("Modélisation et complexité : de la décision à l'action", Revue française de gestion, $n^{\circ} 102$, janvier-février 1995 ; "Stratégie militaire, stratégie d'entreprise : même combat? "Stratégique $n^{\circ} 60,1995 / 4$; "Le rapport à l'autre dans le temps : le jeu et l'action ", in Gestion et théorie des jeux : regards croisés, sous la direction de J. Thépot, Paris, Vuibert, 1998). Elle est chercheur au Centre de Recherche en Économie et Management (CEREM) où ses travaux portent sur le changement dans les entreprises de réseau.
$\mathrm{L}$ ES CONTRAINTES TECHNIQUES DE MISE EN PLACE puis de fonctionnement des infrastructures ont fortement contribué à structurer l'organisation et la culture des entreprises de réseau définies comme des entreprises fournissant des services d'intérêt général à travers l'exploitation d'infrastructures lourdes. Actuellement encore,

« des lignes de communication internes sont mises en place par des compagnies qui mènent, grâce à elles, leur gestion dans une perspective globale, veillant à disposer de capacités disponibles au bon endroit et au bon moment, contrôlent l'utilisation des voies et renseignent le client sur les disponibilités, sur les prix, et sur le cheminement de ce qui a déjà été envoyé. C'est dans ce sens que l'on parle du réseau aérien Air France ou [...] du réseau SNCF. C'est parce qu'il ne saurait y avoir, en certains cas, d'exploitation sans système plus ou moins centralisé de communications internes que la gestion des transports impose l'organisation de ces entreprises spéciales que sont les réseaux » (Claval, 1990).

La prééminence historique de la dimension technique dans le management des entreprises de réseau semble toutefois s'affaiblir avec d'une part les modifications des contraintes techniques propres à l'infrastructure, et d'autre part l'importance croissante d'autres dimensions, notamment institutionnelles, sociales et culturelles, imposées par l'environnement. On peut dès lors se demander si, pour survivre dans un univers désormais turbulent, les entreprises de réseau quittent l'ancien modèle du « château » et adoptent celui du « réseau », pour reprendre les termes de F. Butera (1991).

Toutefois, la notion de réseau est complexe : d'une part les définitions en sont multiples (Curien, 1992 ; Offner et Pumain, 1996), d'autre part le concept peut s'appliquer à divers aspects de l'entreprise (Reich, 1993). Aussi a-t-il semblé préférable d'aborder selon un angle très pragmatique les modalités et les effets du management en réseau à partir d'études de cas. Ces études de cas, qui s'appuient sur l'analyse de documents internes et d'entretiens à la RATP, la SNCF et EDF, montrent que le réseau superpose à la structure dans laquelle il s'inscrit une ou plusieurs dimensions, une dimension étant un sous-ensemble de l'environnement interne ou externe de l'entreprise : géographique, technique, économique, social, commercial. Parce qu'il améliore ou cree des échanges dans les dimensions qu'il favorise, le réseau facilite alors le fonctionnement normal de l'organisation. Si ces dimensions ne servent pas d'enjeux stratégiques, le réseau reste local. Si les enjeux deviennent stratégiques, le réseau est susceptible de servir le changement à l'échelle de l'entreprise. Mais de quel changement s'agit-il ? 


\section{DES RÉSEAUX QUI FACILITENT LOCALEMENT LE MANAGEMENT}

Quatre réseaux locaux seront comparés. Dans un premier temps, on présentera un réseau technique de maintenance des matériels SNCF qui s'est mis en place localement pour répondre à des contraintes de fonctionnement particulières. C'est un réseau qui réussit, sans pour autant avoir vocation à s'étendre au point de remettre en cause l'organisation. Le deuxième cas est aussi un réseau local qui ne remet pas directement en cause l'organisation, et qui renvoie pourtant à un problème stratégique : le réseau d'un « marché » à la SNCF. Les deux derniers réseaux seront présentés ensemble. Leurs finalités sont moins directement liées à des activités opérationnelles puisqu'il s'agit d'un observatoire social et d'un réseau interpersonnel fondé sur une formation commune. Dans la mesure où ils sont mis en place dans des entreprises de réseau, ces réseaux présentent-ils des spécificités?

\section{UN RÉSEAU TECHNIQUE : LA MAINTENANCE DES MATÉRIELS (SNCF)}

L'histoire ferroviaire est marquée par une logique de production, avec comme conséquence un management du réseau et de sa maintenance calqué sur l'organisation physique des infrastructures. Pourtant, il existe dans ce schéma général des réseaux qui surimposent localement des dimensions spécifiques au domaine qu'ils couvrent.

Pour la maintenance des matériels, la logique de management a par exemple superposé à la fois au réseau physique et à la structure organisationnelle correspondante un réseau de cellules réparties sur l'ensemble du territoire qui dialoguent entre elles et sont pilotées par la gestion centrale. Avant cette réorganisation, les responsabilités étaient centralisées à l'échelon de la direction et chaque établissement gérait son parc. Les ateliers étaient peu spécialisés, l'ingénierie de maintenance des séries était déléguée à des centres spécialisés. Depuis le début des années 70, la direction détermine la politique de maintenance, l'évolution du dispositif général de maintenance, l'audit contrôle, la certification, mais elle délègue aux établissements directeurs l'étude de l'optimisation du cycle et des règles de maintenance, ainsi que la gestion courante de sa mise en œuvre. Chaque établissement directeur est coordonnateur de la maintenance d'une série donnée. Il répond à la demande des centres de maintenance de deuxième niveau, chargés de l'entretien rapproché de l'ensemble du matériel et localisés de façon à minimiser la distance entre deux points d'utilisation.

Ainsi, la maintenance des voitures Corail est répartie entre les établissements gérant des voitures et qui ont en charge de faire des opérations de maintenance courantes tandis que d'autres établissements effectuent les opérations lourdes sur ces matériels. Il existe des regroupements par types de matériels voisins et niveaux de maintenance voisins. Les centres de maintenance de deuxième niveau rendent compte directement à la direction de leur gestion et gèrent des informations techniques sur le matériel. Les centres échangent des retours d'expérience par la circulation des informations et des hommes.

Le réseau remplit une fonction bien définie : organiser et gérer la maintenance. Ce réseau allie une infrastructure qui lui est propre (l'implantation des cellules sur le territoire) à des modalités de management fondées sur la délégation et les relations transversales. Les échanges sont de deux sortes. Ceux qui restent malgré tout dans le cadre des relations hiérarchiques descendantes respectent des formes de contrats : la direction délègue des responsabilités aux différents types d'établissements et elle en contrôle la gestion. Les échanges transversaux qui s'effectuent directement entre les centres de premier et deuxième niveau ou qui remontent à la direction sont d'ordre technique, fondés sur une logique de compétence.

Le réseau de la maintenance constitue un cas de déconnexion par rapport au réseau physique qui ne remet pas en cause l'organisation générale. L'évolution s'est effectuée sans pression extérieure, et répond à une volonté de décentralisation ancienne ; le réseau s'intègre dans le fonctionnement de l'ensemble mais de manière adaptée aux contraintes de sa dimension prioritaire : la maintenance selon des niveaux différenciés de matériels complexes.

UN RÉSEAU QUI SURIMPOSE LA DIMENSION COMMERCIALE À LA DIMENSION TECHNIQUE (SNCF)

Le réseau mis en place par le Fret à la SNCF constitue une variante potentiellement plus stratégique du réseau précédent dans la mesure où il favorise une autre dimension que la dimension technique dominante. 
Depuis la création de la SNCF, le réseau d'infrastructure représentait une composante technique très forte et a modelé le management selon une organisation hiérarchique non pas tournée vers l'environnement, mais axée sur la bonne gestion de ce réseau. Le découpage géographique des circonscriptions était complètement conditionné par les voies ferrées. Pour faire face au déplacement du niveau d'arbitrage entraîné par la régionalisation, de nouvelles régions SNCF ont été créées avec la volonté de se rapprocher du découpage administratif. Mais il n'y a toujours pas de région «Centre » calquée sur la région administrative, trop éloignée de la logique du réseau d'infrastructure.

Le Fret, qui concerne les trains de marchandises, a pris une attitude résolument commerciale à la fin des années 80. Il a alors rapidement eu des difficultés pour travailler avec ses clients à partir des structures faites pour l'exploitation et a cherché à avoir une organisation commerciale en phase avec son activité. Un chef d'un « marché " du Fret (un « marché » est une organisation de la SNCF étudiant un secteur d'activité et ayant un portefeuille de clients dans cette activité ; il concerne donc des activités marketing et la vente aux grands comptes) a animé une équipe marketing dont les correspondants dans les régions dépendaient structurellement des directeurs de région. Il a travaillé en réseau avec les agents commerciaux, sans remettre en cause la ligne hiérarchique. Le fonctionnement de ce réseau était reconnu puisqu'il organisait régulièrement des réunions, en plus des multiples communications quotidiennes. Il s'agissait par exemple de fournir aux vendeurs des informations dans un langage commun adapté aux problèmes commerciaux. Il existait aussi des « équipes clients " rassemblant les vendeurs au contact des directions régionales de centrales d'achats. Il fallait en effet une coordination pour homogénéiser les politiques commerciales vis-à-vis des directions régionales. La tutelle hiérarchique des agents commerciaux était informée du fonctionnement du réseau. Ce réseau, fondé sur les compétences, a fonctionné en parallèle avec l'organisation normale, permettant de surimposer une logique commerciale à la logique de production. Il a servi de condensateur d'informations pour les animateurs du réseau, plus au courant du marché que les producteurs eux-mêmes qui n'avaient que peu de contacts entre eux.

Le fonctionnement du réseau dépend de la volonté du gestionnaire du réseau. Il nécessite beaucoup de communication et un objectif clair, de manière à échanger des ressources : en l'occurrence, des informations sur le marché dans un langage commun. Ce cas évoque les conclusions du CNOF (1996) qui se situent implicitement en psychologie sociale dans le cadre de la théorie de la congruence cognitive, selon laquelle les individus interagissent d'autant plus qu'ils partagent les mêmes valeurs, préférences et attributs sociaux, de manière à préserver l'harmonie. Les fonctions du réseau sont ici clairement déterminées par une dimension bien définie (le marketing) et il n'empiète pas sur les prérogatives des régions SNCF. Cependant, il existe parce que l'organisation répond avec retard à une modification de l'environnement, et il existe dans un sous-système se développant selon une logique commerciale en concurrence avec la logique technique qui fonde toujours l'organisation. C'est l'organisation du territoire selon la logique technique qui est remise en cause mais pas le fait que le territoire soit organisé : il faut un découpage différent. D'où la constitution d'un réseau local, opérateur du marché. Mais ce réseau renvoie aussi à l'émergence d'une nouvelle dimension à un niveau décisionnel élevé, et en cela il prend une dimension potentiellement stratégique. Il y a là matière à réflexion en termes de «voisinage " dans la théorie des jeux ${ }^{1}$.

La contrainte technique qui était auparavant stratégique devient une contrainte de coût, donc de moyen. La régionalisation a été utilisée pour modifier l'ensemble du réseau, notamment en créant un échelon intermédiaire (régional) qui puisse prendre en charge les relations avec les régions administratives. La réforme semble actuellement interrompue. Or, la question qui se pose avec de plus en plus d'acuité est de savoir si, dans une logique tirée par le couple produit-client, la structure historique est encore pertinente. D'autant plus que le fret a donné naissance depuis plusieurs années à des organisations dont les succursales empruntent parfois les trains mais ont une gestion indépendante, établissant ainsi une des formes de l'entreprise en réseau de Butera.

\section{DES RÉSEAUX SANS VOCATION DIRECTEMENT OPÉRATIONNELLE}

\section{L'observatoire social, les réseaux interpersonnels fondés sur une formation commune}

D'autres réseaux répondent à des finalités moins directement liées à la production : EDF-GDF, la RATP, la 
SNCF, La Poste ont par exemple mis en place des réseaux d'observation sociale qui ont pour « mission d'éclairer les dirigeants pour une meilleure compréhension du corps social " à EDF-GDF, « d'informer le plus objectivement possible la hiérarchie sur le climat social de la société » à la $\mathrm{SNCF}^{2}$... Ce type de réseau surimpose au fonctionnement hiérarchique normal une nouvelle combinaison de deux dimensions : le corps social qu'il s'agit d'observer d'une part, les décisions des dirigeants qu'il s'agit d'éclairer par une information pertinente d'autre part. Compte tenu de l'autonomie de fonctionnement du réseau qui ne peut être modifiée, l'animateur est impuissant à agir sur un point éloigné : l'information échappe au contrôle du point central initial et devient entièrement dépendante des connexions qui ne respectent pas toujours la ligne hiérarchique. À la différence des réseaux précédents, un problème de valeur d'échange risque d'apparaître, doublé d'un problème de distance.

Le réseau d'observation sociale est toutefois fondé, comme les exemples précédents, sur les compétences. D'autres principes, comme la communauté de formation, peuvent structurer des réseaux interpersonnels. À la SNCF par exemple, les attachés cadres recrutés de façon interne suivent des formations communes de manière à créer un réseau de cadres de l'entreprise plutôt que du transport, de l'équipement ou du matériel. Cette formation lourde, qui altère la hiérarchie des valeurs d'appartenance des cadres, est utilisée de façon consciente. En créant des « trous structuraux $»^{3}$ (White, 1995) et en favorisant des relations « homophiles ${ }^{4}$, elle crée des réseaux interpersonnels qui facilitent le fonctionnement de l'organisation, préparant un changement organisationnel. À la RATP de tels réseaux ont aussi été constitués, accélérant la transition qui succède au changement d'organisation.

\section{COMPARAISON ET ÉVALUATION DES RÉSEAUX}

Les réseaux qui viennent d'être évoqués ont été constitués à partir de dimensions « simples » car bien délimitées : la maintenance, la gestion commerciale, l'observation sociale, la formation. Ils se plaquent sur la structure en place mais, lorsqu'ils rencontrent une dimension émergente, ils peuvent renvoyer à des problèmes stratégiques et devenir susceptibles de contribuer à un changement de grande ampleur. Pour ces réseaux, l'organisation du territoire ne pose pas de problème spécifique : c'est la manière dont l'organisation (et non pas l'infrastructure) modèle les processus de décision et de mise en œuvre qui pose problème et justifie ainsi leur existence.

Les réseaux mobilisent des ressources différentes selon les dimensions concernées : les compétences, le vécu commun au cours des formations. Le fonctionnement s'évalue en termes d'effets de réseaux sur la dimension à partir de laquelle on les observe. Il s'agit de l'apprentissage et de l'efficience technique de l'ensemble des centres dans le cas de la maintenance, de l'information de l'ensemble des membres du réseau dans le cas du « marché » ou de l'observatoire social. En termes de structure, le fonctionnement des réseaux s'évalue par la mise en relation d'autres réseaux fonctionnant selon des logiques différentes : par exemple, les réseaux de la maintenance et $\mathrm{du}$ " marché » mettent en relation le réseau animé par les relations hiérarchiques générales et le réseau des centres de maintenance ou des vendeurs.

Ce type de réseau qui cohabite avec la structure évoque le modèle de la théorie du capital socials ${ }^{5}$ (Lin, 1995). Il engendre toutefois des spécificités de fonctionnement : si le réseau est clairement un « raccourci » en termes d'organisation, il n'engendre pas moins des distorsions de distances (ou de proximités) qui mériteraient d'être étudiées de manière plus approfondie. Par ailleurs, il ne semble pas y avoir de spécificité des entreprises de réseau dans l'apparition et le fonctionnement des réseaux restreints créés pour permettre à une dimension localement dominante de surimposer ses modalités de fonctionnement aux modalités habituelles de l'organisation.

\section{RÉSEAUX ET MANAGEMENT DU CHANGEMENT}

Différents cas de réseaux peuvent être mis en œuvre dans une logique stratégique (Chandler, 1995). À la RATP par exemple, le fonctionnement en réseau a été institué directement comme nouveau mode d'organisation, il est assimilé à la volonté du dirigeant qui l'a établi. Les modalités de cette mise en réseau volontaire contrastent avec le processus de déconnexion de la gestion de l'infrastructure et des services produits sur cette infrastructure à la SNCF. Le changement engendré par ce processus de dissociation de réseaux engendre une rupture stratégique d'un autre ordre que l'instauration du management en réseau à la RATP. 


\section{LE RÉSEAU RÉVOLUTIONNAIRE? LE BOULEVERSEMENT ORGANISATIONNEL À LA RATP}

Depuis sa création officielle, le 1er janvier 1949, la RATP est composée de deux réseaux : réseau ferré, réseau routier. Le fonctionnement du monde souterrain, fermé, de la CMP (Compagnie du Chemin de fer Métropolitain de Paris), est assujetti aux contraintes techniques et de sécurité ; il repose sur une culture d'ingénieur, tandis que le monde ouvert des bus immergés dans la ville de la STCRP (Société des Transports en Commun de la Région Parisienne) a par nature un fonctionnement plus flexible. Par ailleurs, l'espace des négociations dépasse le cadre de l'entreprise. Il met en œuvre des réseaux politiques lorsqu'il s'agit de négocier d'abord la fonction du présidentdirecteur général, mais aussi le budget ou la décision de faire un projet comme METEOR ${ }^{6}$.

Le problème a longtemps été de transformer ces deux réseaux techniques qui coexistent et qui se concurrençaient avant guerre en un réseau de transport unique. La multimodalité a constitué une des étapes des années 80 pour passer à une logique plus intégrée de transport collectif. Les deux mondes sont restés cependant très différents, fusionnés de jure mais pas de facto. Comme l'a résumé Albert David : "d'un point de vue interne comme pour ce qui concerne les interfaces entre la RATP et ses publics, il apparaît clairement (à l'arrivée de Christian Blanc) que les relations sont fortement structurées par des enjeux sociaux et des problèmes techniques mais ont des contenus de gestion très pauvres ${ }^{7}$. En " cassant » les grandes directions et en créant un réseau de seize "départements », Christian Blanc a formellement substitué une nouvelle hiérarchie de dimensions à la hiérarchie précédente.

L'organigramme en râteau qui organise le management en réseau à la RATP est possible dans la mesure où les anciennes directions des travaux neufs et des infrastructures faisaient beaucoup appel à des sociétés extérieures, conservant simplement la maitrise d'ouvrage. De plus, elle est adaptée à la gestion de projets. Par exemple, une fois la décision du projet METEOR prise (décision préparée par le département Développement qui conçoit le projet en fonction des besoins et de la demande de mobilité, en relation avec le design et d'autres départements), sa mise en œuvre dépend de la coordination des départements car le problème est transversal :
- il faut creuser un tunnel ;

- poser des voies ;

- mais aussi faire des stations, donc passer des marchés ;

- comme le métro sera entièrement automatisé, il faut prendre en charge l'électronique (pilotage automatique, signalisation, sécurisation et information du public...) tout en assurant constamment l'interface avec l'ingénierie ferroviaire dont il faut prévoir les contraintes dans le cahier des charges et assurer l'expertise ;

- il faut aussi prévoir le système de péage, de contrôle (sécurité)... toujours en relation avec le design ;

- ainsi que, dès l'origine du projet, les problèmes juridiques de propriété des sous-sols lors des expropriations des particuliers, mais aussi envers la voirie publique ;

- sans oublier la conception de l'utilisation du personnel...

Une équipe Projet avait été constituée pour METEOR. Un département Projet a ensuite été créé, l'infrastructure organisationnelle structurant et pérennisant le fonctionnement en réseau.

Les décisions de Christian Blanc constituent autant de réponses aux remises en cause de la période précédente : pendant la cohabitation, il avait été question de découper l'entreprise dans un processus de privatisation entre le métro, le bus, la maintenance et les bureaux d'études, ce qui avait contribué à déstabiliser le personnel. De plus, le projet EOLE venait d'échapper à la RATP. La structure en râteau et la manière relativement brutale dont elle a été établie a permis de fusionner les mondes du réseau ferré et du réseau routier d'abord en les décomposant (le département bus est dissocié du département ateliers d'entretien des bus, il en est de même pour le métro), mais aussi en individualisant les autres éléments de la chaîne de valeur (projet, développement, commercial... $)^{8}$. Cette modification structurelle accompagne la promotion de la logique de service ${ }^{9}$, élément de stratégie d'une entreprise qui se repositionne explicitement sur un nouveau créneau : « dans les domaines du transport, des espaces et de l'information, nous cherchons à développer les services offerts à nos clients ${ }^{10}$. Enfin, l'organigramme en râteau accompagne la séparation du grade et de la fonction, les deux se justifiant mutuellement car les postes sont alors redistribués en facilitant l'accession à des postes de responsabilité pour un plus grand nombre que dans le système hiérarchique 
précédent. Cependant, quelques années plus tard, les possibilités de promotion sont à nouveau saturées.

Le réseau se décline à l'échelon inférieur par la création de pôles de responsabilité forts, notamment au niveau des unités opérationnelles (voir la Figure 1, p. 36). Jusqu'où va leur autonomie ?

L'équilibre fonctionnel et technique de travail et de responsabilité des chefs de divisions entre métro, RER et bus contribue à gommer les spécificités sans cependant heurter les cultures. Le Comité d'Entreprise Central est décentralisé en CDEP (Comités Décentralisés d'Entreprise). La gestion des œuvres sociales est centrale, mais le traitement de l'organisation du travail reste dans les CDEP. La régulation du temps, qui représente un point très important, est définie dans le détail par le statut du personnel. Les directeurs d'Unités Opérationnelles n'ont pas de pouvoir sur les investissements significatifs comme l'ouverture d'un nouvel accès ou l'achat de matériels lourds. En termes d'organisation, l'accent est mis sur les problèmes du service.

L'institutionnalisation du management en réseau a constitué dans le cas de la RATP un moyen radical (par sa forme et ses modalités de conduite) de prise en main du pouvoir par son nouveau dirigeant. Le management en réseau fait partie d'une stratégie d'ensemble comprenant par ailleurs des négociations politiques sur les moyens et les objectifs de l'entreprise.

\section{LES CONSÉQUENCES STRATÉGIQUES DE LA RECOMPOSITION DES RÉSEAUX : L'EXEMPLE DE LA SNCF}

Si à la RATP il existe un discours fort sur la gestion de la « chaîne de transport " pour « mieux vivre la ville »"1, la structure des activités n'en est pas pour autant fondamentalement remise en cause. À la SNCF au contraire, on assiste à la déconnexion de la gestion de l'infrastructure et des services produits sur cette infrastructure. La chaîne de service autrefois privilégiée cède le pas au client final en termes directement commerciaux appliqués à un service de transport : « le train ».

À la SNCF, l'exemple du Fret illustre la montée de la logique commerciale et les choix de structures qu'elle engendre dans les secteurs connexes. Ainsi la question se pose pour le réseau de maintenance : le matériel doit-il être géré par des entités autonomes ou la prise en compte de la chaîne de valeur conduit-elle plutôt à intégrer le matériel dans la gestion de chacune des activités correspondantes ? Par exemple l'activité Fret pourrait être tentée d'opter pour une organisation qui se démarque de la structure précédente et qui ne soit plus régionale. Une logique de dissociation du Fret, des trains voyageurs grandes lignes et des trains régionaux pose un problème de cohérence dans la gestion des conflits de circulation des différentes catégories de trains.

De manière générale, les différents niveaux hiérarchiques gèrent les trains en accord avec la direction générale. L'optimisation de cette gestion restera de la responsabilité de la SNCF après la réforme portant création du nouvel établissement public RFF (Réseau Ferré de France) qui est chargé de piloter l'évolution du réseau mais qui n'intervient pas dans son exploitation opérationnelle.

La directive européenne de 1991 sur le transport ferroviaire encourage la séparation entre l'infrastructure et le service qui l'emprunte, dans l'optique de favoriser les interpénétrations. Un des défis des opérateurs actuels est de savoir s'ils jouent le jeu de cette concurrence sur le territoire voisin, ou s'ils jouent la coopération (chaîne de valeur). Dans la pratique, des contraintes techniques gênent la circulation des trains :

- l'hétérogénéité des technologies : les réseaux européens ont développé des principes et des technologies de signalisation et de sécurité différents et pas nécessairement compatibles (non-interopérabilité) ;

- l'hétérogénéité des matériels : c'est une conséquence de l'hétérogénéité des technologies, car le matériel est lié à l'infrastructure. La signalisation au sol a sa partie embarquée cohérente, les types de courant électriques sont parfois différents, les caractéristiques des caténaires aussi.

On peut imaginer, à défaut d'entreprises qui couvrent la production et la commercialisation de manière transeuropéenne, une fusion sur la partie commerciale en conservant une gestion géographique de la production (voir la Figure 2, p. 36).

Des solutions de ce type existent déjà sous différentes formes : filiales communes, GIE (Groupements d'Intérêt Économique) européens, ou accords commerciaux. 
Eurostar par exemple est géré sous ce régime. En Fret, il existe un système comparable de services de trains de marchandises entre la Suède et l'Italie du Nord, comprenant des structures de pilotage communes et des accords avec chaque réseau traversé pour l'exécution du transport. Cette logique est fondée sur la vente d'un produit particulier qui est un service de transport, appelé train. L'entité commune conçoit le produit et en assure la commercialisation, les responsables des réseaux traversés s'engagent quant à eux à la réalisation avec la qualité, la sécurité et la régularité convenues.

À la différence de la RATP où le management du changement par une organisation en réseau relevait d'une stratégie de fusion de deux réseaux historiquement et techniquement différenciés, la CMP et la STCRP, la problématique d'entreprises de réseau comme les transports ferroviaires et l'électricité consiste à dissocier les réseaux sur lesquels leurs activités sont fondées, bouleversant ainsi profondément leur univers de management notamment. La recomposition des réseaux est toutefois subordonnée à des choix politiques, qu'il s'agisse du réseau électrique comme du réseau ferré. En ce qui concerne le choix des péages par exemple, les négociations européennes en cours aussi bien que les considérations nationales de service public pèsent sur les discussions entre la SNCF et le gouvernement français. La montée en puissance de la logique client ou commerciale (ou de produit), qui se superpose à la logique du réseau physique (infrastructure) et à celle du réseau géographique ou institutionnel (région) engendre une dynamique d'évolution qui nécessitera des arbitrages pouvant conduire à des recompositions des relations entre les différents réseaux.

\section{CONCLUSION : LES SPÉCIFICITÉS DE MANAGEMENT DES ENTREPRISES DE RÉSEAU}

La logique commerciale a des limites : dans la mesure où le réseau est un système de couverture d'un territoire qui renvoie à la fonction assurée (réseau-opérateur), il est susceptible de fournir des « services d'intérêt économique général ». La communication de la commission européenne de 1996 les définit ainsi : « mentionnés dans le traité à l'article 90, ils désignent les activités de service marchand remplissant des missions d'intérêt général, et soumises de ce fait par les États membres à des obligations spécifiques de service public. C'est le cas en particulier des services en réseaux de transport, d'énergie, de communication »12.

Si les réseaux sont recomposés, les fonctions qu'ils assurent diffèrent : à la Poste, le regroupement des facteurs modifiera le rôle du bureau de poste. Réciproquement, l'évolution des missions d'intérêt général est susceptible de réagir sur la gestion des réseaux. Ainsi la modification de la demande de transport sur laquelle le réseau de la RATP était structuré13 a des conséquences sur la politique de l'entreprise. Il fallait répondre à la demande de transport centre-périphérie, périphériecentre en heure de pointe. Désormais, les gens se déplacent pour d'autres motifs que le travail (faire ses courses, aller au cinéma, au musée...). Pour les trajets quotidiens, la demande concerne désormais un service de sécurité, des informations en cas de perturbation, une qualité du service de transport en général. Pour le transport en dehors des heures de pointe, le besoin porte sur de l'information (les itinéraires, les tarifs) et de « l'assistance » (poussettes). Il y a en effet une diversification du public, qui passe d'une société relativement homogène à très hétérogène. Du coup, une partie du travail s'apparente à un service social de fait, mais jusqu'à quel point ? La superposition des entreprises de réseau aux territoires et aux problèmes de société renvoie ainsi à la fonction sociale des entreprises de réseau à travers la dimension "service public », elle-même reliée à la dimension « intérêt général ». Le développement local (services de proximité, relations avec les élus, les associations locales...) interagit avec la gestion des entreprises de réseau.

Trois remarques s'imposent alors. Sur le rôle de l'inscription physique des infrastructures dans l'espace tout d'abord. En effet, les contraintes techniques de gestion des interfaces (structure du réseau technique), autrefois prioritaires, s'estompent avec l'accroissement de la capacité de traitement des données et l'apparition des nouveaux réseaux de communication qui modifient les conditions de la gestion. De plus, le service n'est pas le réseau lui-même mais des services qui lui sont associés. Le développement des nouvelles conceptions du produit remplace le rôle des contraintes techniques dans la stratégie de l'entreprise, appelant et justifiant à la fois des modalités de management en réseau. Enfin, ce ne sont pas les modalités de l'éventuelle gestion en réseau qui les différencient des autres entreprises : si l'on prend en compte uniquement la dimension économique, les 
réseaux de management ne présentent pas de spécificités dans leur mise en œuvre ou leur utilisation. Par contre, les entreprises de réseau ont des éléments de contexte commun, notamment parce que la notion de service d'intérêt général introduit dans l'univers décisionnel de l'entreprise des enjeux politiques qui le dépassent. Les logiques qui coexistent ou s'opposent alors au sein des entreprises de réseau dépendent notamment de la définition par l'État des missions d'intérêt général qui déterminent leurs obligations spécifiques de service public.
Selon leur importance du moment et les conditions de l'environnement, ces différentes logiques influent sur les modalités de management. Le management constitue ainsi un point névralgique dans l'adaptation des entreprises de réseau à l'évolution du concept d'opérateur unique. Les réseaux apparaissent alors comme un moyen de management transitoire, qui n'est dédié à aucune finalité en particulier : selon les circonstances, il sert le maintien de l'organisation en l'état ou le changement de l'organisation en vue du maintien de l'entité.
1. Voir G. Umbauher, 1998.

2. J. Y. Mareau, cité par Philippe Baverel, Le Monde, 29 janvier 97. Les formulations des deux citations expriment des sensibilités un peu différentes.

3. Un trou structural est un vide entre contacts non redondants (Burt, 1995). Deux contacts sont redondants lorsqu'ils procurent le même bénéfice en information. On mesure la redondance par :

- la cohésion (des contacts fortement connectés les uns aux autres apportent vraisemblablement les mêmes informations, procurant ainsi les mêmes bénéfices),

- l'équivalence structurale (ce sont des contacts qui, indépendamment des relations qu'ils ont entre eux, relient un manager à des tierces parties : ces contacts ont les mêmes sources d'information et procurent les mêmes bénéfices).

4. Interactions sociales ayant lieu entre individus de niveaux hiérarchiques semblables.
5. Un auteur comme N. Lin définit le capital social comme "l'investissement d'un individu dans ses relations avec d'autres" (Lin, 1995). Voir l'analyse des liens entre réseau et théorie du capital social dans A. Marchais-Roubelat, 1997.

6. Voir l'analyse détaillée d'Albert David (A. David, 1995).

7. Ibid., p. 15.

8. Ibid., p. 28-29.

9. Cette notion n'est pas nouvelle à la RATP : Claude Quin l'avait déjà soutenue lors de sa présidence.

10. A. David, op. cit. p. 54.

11. Plan d'entreprise de la RATP, 1995-97.

12. Communication de la commission. Les services d'intérêt général en Europe, Commission européenne, 1996, p. 6.

13. E. Heurgon, La RATP pour "Mieux Vivre la Ville", Rencontres thématiques de l'ENSPTT, vendredi 14 juin 1996.

\section{BIBLIOGRAPHIE}

Bouttes J. P., LeBAN R. 1994. Concurrence et réglementation dans les industries de réseau en Europe. Du cas général à celui de l'électricité. CEREM.

BURT R. S. 1995. « Le capital social, les trous structuraux et l'entrepreneur ». Revue française de sociologie, $\mathrm{n}^{\circ} 36-4$, octobre-décembre, pp. 599-628.

BUTERA F. 1991. La métamorphose de l'organisation, Du château au réseau. Paris, Éditions d'Organisation. Version originale : 1983.

Chander A. 1995. «L'évolution historique de la grande entreprise industrielle : structures et capacités organisationnelles ». Entreprises et histoire, ${ }^{\circ} 10$, décembre, pp. 13-19.

Claval P. 1990. «La mise en réseau des territoires ». In Communications et territoires, sous la direction de $\mathbf{H}$. Bakis, Paris, La Documentation Française.

CNOF. 1996. Management France. ${ }^{\circ} 95$.

CURIEN N. (dir). 1992. Économie et Management des entreprises de réseau. Économie des réseaux, réseaux organisateurs, management en réseau. Paris, Economica.

David A. 1995. RATP. La métamorphose. Réalités et théorie du pilotage du changement. Paris, InterEditions.
GDR RÉSEAUX. 1995. Rapport d'activités 1992-1995. Noisy-le Grand.

HeURgon E. 1996. La RATP pour "Mieux Vivre la Ville». Rencontres thématiques de l'ENSPTT.

LIN N. 1995. « Les ressources sociales : une théorie du capital social ». Revue française de sociologie $\mathrm{n}^{\circ} 36-4$, octobredécembre, pp. 685-704.

Marchais-Roubelat A. 1997. «Peut-on gérer en réseau les entreprises de réseau ? » CEREM.

Offner J. M., Pumain D. (dir). 1996. Réseaux et territoires, significations croisées. La Tour d'Aigues, Éditions de l'Aube.

ReICH R. 1993. L'économie mondialisée. Paris, Dunod.

UMBhauer G. 1998. "Jeux évolutionnistes et concept de voisinage : une application à l'évolution des réseaux ». In Approches multiformes de la proximité, sous la direction de M. Bellet, T. Kirat, C. Largeron. Paris, Hermès.

White H. 1995. «Passages réticulaires, acteurs et grammaire de la domination », Revue française de sociologie $\mathrm{n}^{\circ} 36-4$, octobre-décembre, pp. 705-723. 